\title{
Pulmonary Perfusion Changes as Assessed by Contrast-Enhanced Dual-Energy Computed Tomography after Endoscopic Lung Volume Reduction by Coils
}

\author{
Frédéric Lador ${ }^{\mathrm{a}}$ Anne-Lise Hachulla ${ }^{\mathrm{b}}$ Olivia Hohn ${ }^{\mathrm{c}}$ Jérôme Plojoux ${ }^{\mathrm{a}}$ \\ Maxime Ronot ${ }^{d-f}$ Xavier Montet $^{\mathrm{b}}$ Paola M. Soccal ${ }^{\mathrm{a}}$ \\ a Service of Pulmonary Medicine, ${ }^{\mathrm{b}}$ Division of Radiology, Geneva University Hospitals, and ${ }^{\mathrm{C}}$ Geneva Faculty of \\ Medicine, Geneva, Switzerland; 'Department of Radiology, APHP, University Hospitals Paris Nord Val de Seine, \\ Beaujon Hospital, Clichy, and 'University Paris Diderot, Sorbonne Paris Cité, and fINSERM U1149, Centre de \\ Recherche Biomédicale Bichat-Beaujon, CRB3, Paris, France
}

\section{Keywords}

Pulmonary perfusion - Lung volume reduction by coils . Dual-energy computed tomography

\section{Abstract}

Background: Endoscopic lung volume reduction by coils (LVRC) is a recent treatment approach for severe emphysema. Furthermore, dual-energy computed tomography (DECT) now offers a combined assessment of lung morphology and pulmonary perfusion. Objectives: The aim of our study was to assess the impact of LVRC on pulmonary perfusion with DECT. Methods: Seventeen patients $(64.8 \pm 6.7$ years) underwent LVRC. DECT was performed prior to and after LVRC. For each patient, lung volumes and emphysema quantification were automatically calculated. Then, 6 regions of interest (ROls) on the iodine perfusion map were drawn in the anterior, mid, and posterior right and left lungs at 4 defined levels. The $\mathrm{ROI}$ values were averaged to obtain lung perfusion as assessed by the lung's iodine concentration $\left(C_{\text {Lung, }} \mu \mathrm{gg} \cdot \mathrm{cm}^{-3}\right)$. The $C_{\text {Lung }}$ values were normalized using the left atrial iodine concentration $\left(C_{\mathrm{LA}}\right)$ to take into account differences between successive DECT scans. Results: The 6-min walk distance (6MWD) improved significantly after the procedure ( $p=0.0002)$. No lung volume changes were observed between successive DECT scans for any of the patients ( $p=0.32)$, attesting the same suspended inspiration. After LVRC, the emphysema index was significantly reduced in the treated lung $(p=0.0014)$. Lung perfusion increased significantly adjacent to the treated areas $\left(C_{\text {Lung }} / C_{\mathrm{LA}}\right.$ from 3.4 \pm 1.7 to $5.6 \pm 2.2, p<0.001)$ and in the ipsilateral untreated areas (from $4.1 \pm 1.4$ to $6.6 \pm 1.7, p<0.001$ ), corresponding to a mean 65 and $61 \%$ increase in perfusion, respectively. No significant difference was observed in the contralateral upper and lower areas (from $4.4 \pm 1.9$ to $4.8 \pm 2.1, p=0.273$, and from $4.9 \pm 2.0$ to $5.2 \pm 1.7, p=0.412$, respectively). A significant correlation between increased 6MWD and increased perfusion was found $\left(p=0.0027, R^{2}=0.3850\right)$. Conclusions: Quantitative analysis based on DECT acquisition revealed that LVRC results in a significant increase in perfusion in the coil-free areas adjacent to the treated ones, as well as in the ipsilateral untreated areas. This suggests a possible role for LVRC in the improvement of the ventilation/perfusion relationship.

(c) 2016 S. Karger AG, Basel

\section{KARGER}

E-Mail karger@karger.com

www.karger.com/res
C 2016 S. Karger AG, Basel

0025-7931/16/0926-0404\$39.50/0

F.L. and A.-L.H. contributed equally to this work.

Anne-Lise Hachulla, MD

Division of Radiology, Geneva University Hospitals

Rue Gabrielle-Perret-Gentil 4

CH-1211 Geneva 14 (Switzerland)

E-Mail Anne-lise.hachullalemaire@ hcuge.ch 


\section{Introduction}

Lung volume reduction surgery (LVRS) and lung transplantation are recognized options for the treatment of patients with severe emphysema. However, their use is limited, since only selected patients are eligible. The latter procedure is hampered by a shortage of organs $[1$, 2 ], while the former method has been associated with morbidity and mortality, making its attractiveness debatable $[3,4]$. Hence, new treatment modalities are desirable for this category of patients, who may already benefit from improvement of symptoms with pulmonary rehabilitation or medical pharmacological treatments $[5,6]$. Consequently, endoscopic lung volume reduction by coils (LVRC) has shown benefits especially for patients with severe emphysema and hyperinflation $[7,8]$, with a satisfying safety profile and long-lasting results [9-11].

Whilst the symptomatic improvement after LVRC is attributed to the impact of the procedure on pulmonary function tests, little is known about its effect on lung perfusion. In fact, it may be that the volume reduction is also accompanied by a redistribution of pulmonary blood flow towards better-ventilated areas. As a consequence, LVRC may also improve the pulmonary ventilation/perfusion ratio. To the best of our knowledge, however, lung perfusion changes after LVRC have not been studied so far.

Dual-energy computed tomography (DECT) is a technology recently made available which allows conducting a combined functional and morphological analysis of the lung. DECT images are obtained by single acquisition using $2 \mathrm{X}$-ray tubes or rapid peak voltage switching [12]. These acquisitions permit material differentiation according to 3-material decomposition mathematical algorithms. Because of the attenuation properties of iodine at 2 different photon energies ( 80 and $140 \mathrm{kV}$ ), the materialspecific contrast-enhanced DECT technique can generate lung perfusion maps by quantifying the iodine concentration in the parenchyma [13]. In comparison with conventional CT, no additional cost, extra radiation dose, or extra intravenous iodine contrast medium injection is needed. Functional image processing is simply added, offering an invaluable and simultaneous exploration of the vascular anatomy, parenchymal morphology, and functional perfusion [14-16].

The aim of this study was to assess the impact of LVRC on pulmonary perfusion with DECT.

\section{Methods}

Over an 18-month period (September 2013 to December 2015), 17 patients $(64.8 \pm 6.7$ years, BMI $23.8 \pm 3.9)$ with severe emphysematous disease underwent a total of 25 LVRC procedures at our institution and took part in the study; 9 patients underwent a unilateral procedure, and 8 patients a consecutive bilateral procedure within a 3 -month period. Twelve LVRC procedures were done in the right upper lobe, 8 in the left upper lobe, 2 in the right lower lobe, and 2 in the left lower lobe. The patients were selected according to the recommended inclusion and exclusion criteria (Table 1) for LVRC [17]. All cases were discussed during a multidisciplinary board meeting including pneumologists, radiologists, and thoracic surgeons. All patients gave informed written consent, and the protocol was approved by the local institutional review board.

\section{Description of the LVRC Procedure}

The selected patients underwent bronchoscopy, during which 10-12 coils were inserted into the selected pulmonary lobe. The implantation site was determined after a CT evaluation of the emphysematous topography. The mean procedure time was around $45 \mathrm{~min}$. Only one lung was treated at once; after a recovery time of at least 3 months after the intervention, coils were placed in the contralateral lung, thus achieving bilateral treatment.

The equipment for the procedure consists of a flexible bronchoscope, a guidewire, a catheter, forceps, a cartridge, and coils. The coils (RePneu; PneumRx, Düsseldorf, Germany) are made of shape-memory nitinol wire, which behaves like spiral springs. The length is chosen according to the size of the target airway; 100, 125, and $150 \mathrm{~mm}$ are the 3 available lengths. During the procedure, a standard bronchoscope is directed into the target airway. The guidewire is advanced first and positioned $15-20 \mathrm{~mm}$ from the pleura. The catheter is advanced over the guidewire and aligned with its distal tip. The length of the airway is measured with radiopaque markers located on the guidewire to determine the appropriate length of the coil. After removal of the guidewire, a straightened coil, preloaded in a cartridge, is pushed through the catheter with the forceps. The coil is then deployed by removal of the catheter under fluoroscopic guidance. After deployment, the coil reassumes its curved shape, and if its position is satisfactory, the coil is released in the lung parenchyma. If necessary, it is possible to reposition or remove the coil. All procedures were performed under general anesthesia, and fluoroscopy was used to confirm the coil's position.

\section{Assessment and Follow-Up}

Data were collected before the first intervention, including functional test $\left(\mathrm{FEV}_{1}\right.$ [forced expiratory volume in the 1st second], FVC [forced vital capacity], TLC [total lung capacity], RV [residual volume], FRC [functional residual capacity], and blood gas analysis) and contrast-enhanced DECT quantitative data (see below). Exercise capacity was evaluated by 6 -min walk distance (6MWD). Each of the parameters was measured again after each intervention to follow and assess the effect of LVRC.

DECT Technique

All of the 17 patients underwent DECT acquisition before and 3 months after each procedure. For each of the patients, the same protocol was applied on the same DECT scanner. 
Table 1. Inclusion and exclusion criteria

\begin{tabular}{ll}
\hline Inclusion criteria & Exclusion criteria \\
\hline$\geq 35$ years of age & DLCO $<20 \%$ of predicted \\
Postbronchodilator $\mathrm{FEV}_{1} \leq 45 \%$ of predicted & $6 \mathrm{MWD}<140 \mathrm{~m}$ \\
Total lung capacity $>100 \%$ of predicted & History of recurrent respiratory failures \\
Residual volume $>175 \%$ of predicted & Pulmonary hypertension $>50 \mathrm{~mm} \mathrm{Hg}^{1}$ \\
Dyspnea $\geq 2(\mathrm{mMRC})$ & Bullae in $>1 / 3$ of the lung \\
Stopped smoking for $\geq 8$ weeks & Previous LVRS, lung transplant, or lobectomy \\
Bilateral emphysema $(\mathrm{CT})$ & Patient on $>20$ mg prednisone a day \\
& Patient is on an antiplatelet agent (such as clopidogrel) or on \\
& anticoagulant therapy (such as with heparin or Coumadin) or \\
& has not been weaned off prior to the procedure
\end{tabular}

DLCO, diffusing capacity for $\mathrm{CO} ; \mathrm{FEV}_{1}$, forced expiratory volume in the 1 st second; $6 \mathrm{MWD}$, 6-min walk distance; mMRC, modified medical research council dyspnea scale; LVRS, lung volume reduction surgery.

${ }^{1}$ Pulmonary artery systolic pressure estimated by transthoracic echocardiography.

Twelve patients were scanned with a second-generation dualsource 128-slice multiple detector CT system (Somatom Definition Flash; Siemens Healthcare, Forchheim, Germany). The acquisition protocol was as follows: scan parameters of $80 \mathrm{kVp}$ and thinfiltered (Sn) $140 \mathrm{kVp}$ at an effective 300 and $100 \mathrm{mAs}$, respectively; collimation $128 \times 0.6 \mathrm{~mm}$; rotation time $0.28 \mathrm{~s}$; and pitch 0.55 . The 5 remaining patients underwent DECT on a dual-energy gemstone spectral CT device (Discovery CT 750 HD; GE Healthcare, Milwaukee, WI, USA). The acquisition protocol was as follows: 80 and $140 \mathrm{kV}$ tube voltage with fast switched spectral CT in gemstone spectral imaging mode; $630 \mathrm{~mA}$ tube current; collimation $64 \times$ $0.625 \mathrm{~mm}$; rotation time $0.6 \mathrm{~s}$; and pitch 0.984 .

All CT scans were performed at suspended full inspiration after intravenous administration of $80 \mathrm{~mL}$ of iohexol (350 mg of iodine/ $\mathrm{mL}$; GE Healthcare, Switzerland) at a flow rate of $4 \mathrm{~mL} / \mathrm{s}$ followed by $50 \mathrm{~mL}$ of saline flush. A region of interest (ROI) was drawn in the ascending aorta, and imaging acquisition started $5 \mathrm{~s}$ after a threshold of 100 Hounsfield units (HU) was reached. The acquisition was performed in the caudocranial direction. The effective radiation dose was estimated by using the dose-length product and a conversion coefficient $(\mathrm{k}=0.014 \mathrm{mSv} /[\mathrm{mGy} \cdot \mathrm{cm}])[16]$.

\section{Imaging Analysis}

Quantitative Morphological Analysis

Parenchymal and mediastinal reconstructions were performed for the morphological analysis. For each DECT scan, the right and left lungs and lobes were automatically segmented using dedicated software (Pulmo3D ${ }^{\circledR}$, on a Syngovia VA30 Multimodality Workplace; Siemens Medical Solutions, Forchheim, Germany). The low-attenuation density threshold for emphysema quantification was $-50 \mathrm{HU}$, as recommended in the literature $[18,19]$. Lung and lobe volumes (in liters) and the emphysema index (defined as the percent area of the lung with attenuation values below -950 HU) were automatically calculated.

Quantitative Perfusion Analysis

Iodine perfusion maps were generated using a dedicated workstation depending on the CT protocol, and normalized by en- hancement of the left atrium (Syngovia Multimodality Workplace; Siemens Medical Solutions or GE workstation, VolumeShare 5, GE Healthcare, USA). For the lung perfusion study, the parenchymal iodine concentration $\left(\mathrm{C}_{\text {Lung }}, \mu \mathrm{g} \cdot \mathrm{cm}^{-3}\right)$ was measured. For each patient, 6 ROIs were drawn in the anterior, mid, and posterior areas of the right and left lungs at 4 defined levels (1: aortic arch; 2: carina; 3: right pulmonary artery; and $4: 2 \mathrm{~cm}$ above the diaphragm). The ROIs were made as large as possible, but avoiding coils, consolidation, or ground-glass opacities. All $\mathrm{C}_{\text {Lung }}$ values were normalized using the left atrial iodine concentration $\left(\mathrm{C}_{\mathrm{LA}}\right.$, $\mu \mathrm{g} \cdot \mathrm{cm}^{-3}$ ) to take into account possible differences between successive DECT acquisitions. Finally, the $\mathrm{C}_{\mathrm{Lung}} / \mathrm{C}_{\mathrm{LA}}$ measurements were grouped for each patient according to their anatomical distribution (pulmonary areas) into 4 areas: (1) adjacent to treated areas; (2) ipsilateral untreated areas; (3) contralateral upper areas; and (4) contralateral lower areas, as illustrated in Figure 1.

\section{Statistical Analysis}

Normal distribution of the data was assessed through a Kolmogorov-Smirnov test. Values are expressed as means (SD) or medians (ranges) according to the distribution of the variable, and percentages, as appropriate. Data are given as means \pm SD unless otherwise stated. Continuous data comparisons were performed with the Wilcoxon matched-pairs test. Two-tailed analysis was always used. A $p$ value $<0.05$ was considered to be statistically significant. All statistical analyses were performed using SPSS 20.0 (SPSS Inc., Chicago, IL, USA).

\section{Results}

\section{Functional Tests and Exercise Capacity}

The results of the pulmonary function tests, blood gas analyses, and 6MWD tests before and after the LVRC procedure are reported in Table 2. We observed a global trend toward improvement. RV was the only parameter 
Fig. 1. a Illustration of the 4 lung areas: (1) adjacent to treated areas; (2) ipsilateral untreated areas; (3) contralateral upper areas; and (4) contralateral lower areas. b Four defined levels (1: aortic arch; 2: carina; 3: right pulmonary artery; and $4: 2 \mathrm{~cm}$ above the diaphragm). c Six ROIs drawn in the anterior (ANT), mid (LAT), and posterior (POST) areas of the right and left lungs.
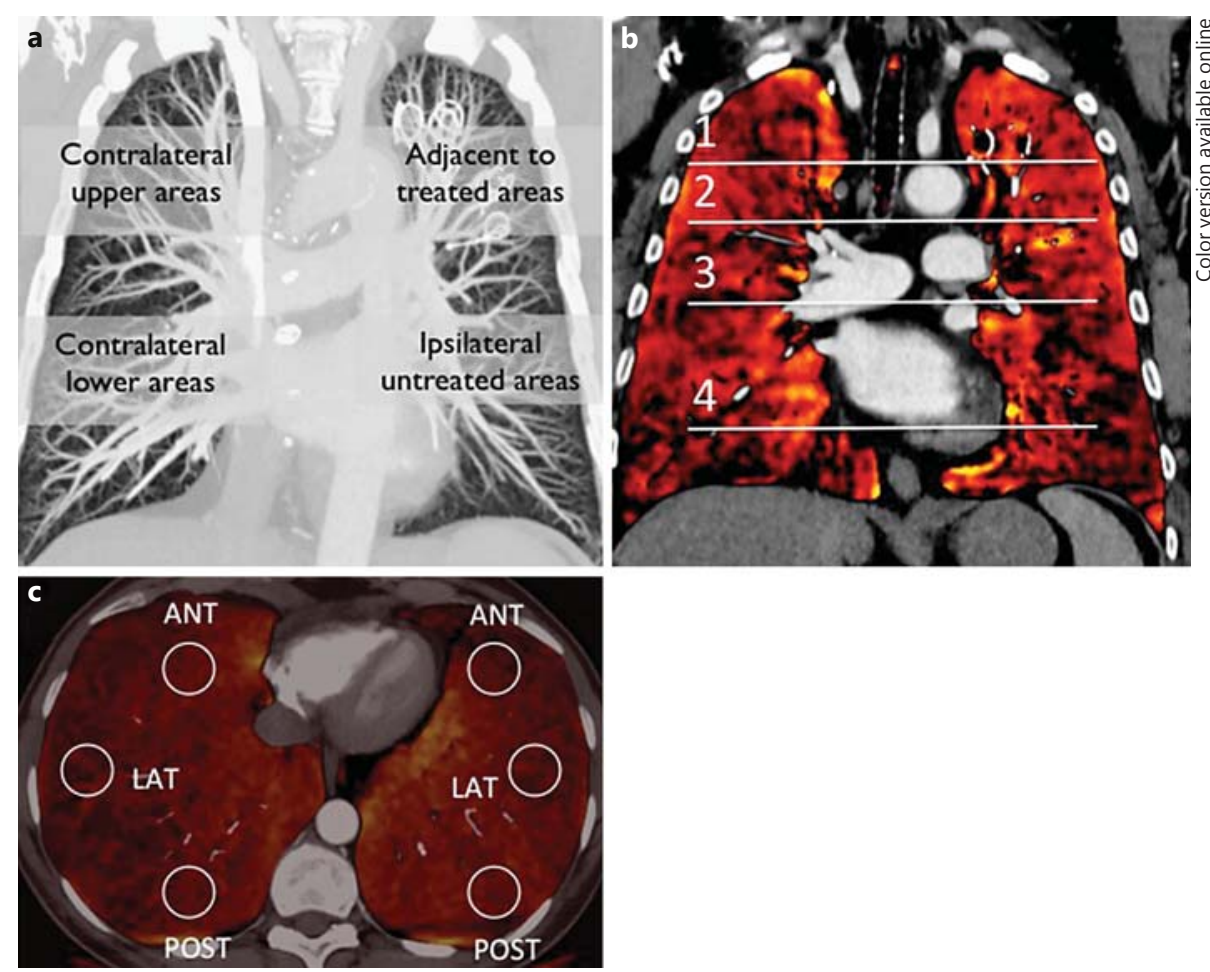

Table 2. Pulmonary function tests, blood gas analysis, and 6MWD before and after LVRC

\begin{tabular}{lccc}
\hline & $\begin{array}{l}\text { Before } \\
\text { procedure }\end{array}$ & $\begin{array}{l}\text { After } \\
\text { procedure }\end{array}$ & $p$ value \\
& $0.91 \pm 0.28$ & $0.97 \pm 0.35$ & $\mathrm{~ns}$ \\
$\mathrm{FEV}_{1}, \mathrm{~L}$ & $2.88 \pm 0.78$ & $2.97 \pm 0.73$ & $\mathrm{~ns}$ \\
$\mathrm{FVC}, \mathrm{L}$ & $7.53 \pm 1.29$ & $7.19 \pm 0.81$ & $\mathrm{~ns}$ \\
$\mathrm{TLC}, \mathrm{L}$ & $4.58 \pm 0.99$ & $4.14 \pm 0.65$ & $<0.05$ \\
$\mathrm{RV}, \mathrm{L}$ & $153 \pm 18$ & $145 \pm 24$ & $\mathrm{~ns}$ \\
$\mathrm{RV} / \mathrm{TLC}, \%$ predicted & $5.72 \pm 1.18$ & $5.42 \pm 0.84$ & $\mathrm{~ns}$ \\
$\mathrm{FRC}, \mathrm{L}$ & $8.19 \pm 1.50$ & $8.25 \pm 0.99$ & $\mathrm{~ns}$ \\
$\mathrm{PaO}_{2}, \mathrm{kPa}$ & $4.63 \pm 1.62$ & $4.68 \pm 1.21$ & $\mathrm{~ns}$ \\
$\mathrm{~A}-\mathrm{a} \mathrm{O} \mathrm{O}_{2}$ gradient, kPa & $340 \pm 102$ & $408 \pm 76$ & 0.0002 \\
$6 \mathrm{MWD}^{2} \mathrm{~m}$ & & & \\
\hline
\end{tabular}

$\mathrm{FEV}_{1}$, forced expiratory volume in the 1st second; FVC, forced vital capacity; TLC, total lung capacity; RV, residual volume; FRC, functional residual capacity; 6MWD, 6-min walk distance; ns, not significant.

that significantly decreased $(p<0.05)$. The arterial oxygen pressure tended to increase, whereas the alveolar-arterial gradient remained stable. The $6 \mathrm{MWD}$ had improved significantly after the procedure compared to before $(p=$ 0.0002).

Pulmonary Perfusion Changes after LVRC

\section{Quantitative Morphological Image Analysis}

A total of 42 DECT scans (corresponding to a mean number of CT scans of 2.5 per patient) were performed. No lung volume changes were observed between successive DECT scans for any of the patients $(p=0.32)$, attesting the same suspended inspiration during the acquisitions.

After the LVRC procedure, the emphysema index was significantly reduced in the treated lung $(p=0.0014)$, but no significant difference was calculated for the contralateral untreated lung $(p=0.7)$. All morphological quantification values are detailed in Table 3 .

\section{Quantitative Perfusion Image Analysis}

After LVRC, perfusion increased significantly adjacent to the treated areas $\left(\mathrm{C}_{\text {Lung }} / \mathrm{C}_{\mathrm{LA}}\right.$ from $3.4 \pm 1.7$ to 5.6 $\pm 2.2, p<0.001)$ and in the ipsilateral untreated areas (from $4.1 \pm 1.4$ to $6.6 \pm 1.7, p<0.001$ ), corresponding to a mean $65 \pm 29$ and $61 \pm 21 \%$ increase in perfusion, respectively. No significant perfusion changes were observed in the contralateral upper and lower areas (from $4.4 \pm 1.9$ to $4.8 \pm 2.1, p=0.273$, and from $4.9 \pm 2.0$ to 5.2 $\pm 1.7, p=0.412$, respectively) (Fig. 2). Table 4 shows the results for the unilateral and bilateral procedures separately. 
Table 3. Results of the quantitative morphological image analysis before and after LVRC

\begin{tabular}{lccc}
\hline & Before procedure & After procedure & $p$ value $^{1}$ \\
\hline Lung volume, L & $6.7 \pm 0.9$ & $6.6 \pm 0.9$ & 0.32 \\
\hline Volume of the treated lung, L & $3.4 \pm 0.4$ & $3.3 \pm 0.4$ & 0.12 \\
Emphysema index of the treated lung, \% & $25.1 \pm 12$ & $20.7 \pm 8$ & 0.001 \\
\hline Volume of the treated lobe, L & $1.7 \pm 0.4$ & $1.6 \pm 0.4$ & 0.006 \\
Emphysema index of the treated lobe, \% & $31.8 \pm 17$ & $25.3 \pm 12$ & $<0.0001$ \\
\hline Volume of the ipsilateral untreated lobes, L & $1.6 \pm 0.3$ & $1.6 \pm 0.3$ & 0.24 \\
Emphysema index of the ipsilateral untreated lobes, \% & $15.7 \pm 7.3$ & $15.0 \pm 7.2$ & 0.18 \\
\hline Volume of the contralateral lung, L & $3.3 \pm 0.5$ & $3.4 \pm 0.5$ & 0.41 \\
Emphysema index of the contralateral lung, \% & $23.0 \pm 8.9$ & $23.3 \pm 8.8$ & 0.79 \\
\hline
\end{tabular}

Values are expressed as means $\pm \mathrm{SD}$.

${ }^{1} p$ value comparing the score before with that after the procedure.

Table 4. Results of pulmonary perfusion before and after LVRC for patients after unilateral versus those after bilateral procedures

\begin{tabular}{lllc}
\hline & Before procedures & After procedures & $p$ value $^{1}$ \\
\hline Patients after unilateral procedures & & & $<0.0001$ \\
$\quad$ Adjacent to treated areas & $3.3 \pm 1.5$ & $5.5 \pm 2.3$ & 0.0001 \\
Ipsilateral untreated areas & $4.1 \pm 1.5$ & $6.3 \pm 1.7$ & 0.32 \\
Contralateral upper areas & $4.1 \pm 1.8$ & $3.9 \pm 1.7$ & 0.18 \\
$\quad$ Contralateral lower areas & $4.1 \pm 1.5$ & $4.4 \pm 1.4$ & 0.008 \\
Patients after bilateral procedures & & & 0.006 \\
Adjacent to first treated areas & $3.9 \pm 2.2$ & $6.6 \pm 1.9$ & 0.008 \\
First ipsilateral untreated areas & $4.7 \pm 1.9$ & $7.2 \pm 1.6$ & 0.0007 \\
Adjacent to second treated areas & $3.4 \pm 1.7$ & $5.7 \pm 1.7$ & $7.1 \pm 1.8$ \\
Second ipsilateral untreated areas & $4.3 \pm 1.9$ & &
\end{tabular}

Pulmonary perfusion values are expressed as $\mathrm{C}_{\text {Lung }} / \mathrm{C}_{\mathrm{LA}}$. Values are expressed as means $\pm \mathrm{SD}$.

${ }^{1} p$ value comparing the score before with that after the procedures.

Figure 3 shows an example of morphological CT scans and iodine perfusion maps obtained before (Fig. 3a, lower panel) as well as after the first (Fig. 3b, lower panel) and the second (Fig. 3c, lower panel) LVRC. It allows visually appreciating the increment of perfusion in the lung parenchyma adjacent to the coils and in the ipsilateral, untreated area (Fig. 4). In Figure 5, the percent change in global lung perfusion is shown against the percent change in 6MWD. A significant correlation between increased $6 \mathrm{MWD}$ and increased perfusion was found $(p=0.0027$, $\left.R^{2}=0.3850\right)$.

The mean dose-length product was $294 \pm 97 \mathrm{mGy} \cdot \mathrm{cm}$, corresponding to a mean effective dose of $4.1 \pm 1.3 \mathrm{mSv}$.

\section{Discussion}

The current pilot study aimed at evaluating the impact of LVRC on lung perfusion and pulmonary function tests. We observed a significant increase in perfusion adjacent to the treated areas and in the ipsilateral untreated areas, whereas the perfusion in the contralateral upper and lower lobes remained stable.

Similar to what has been demonstrated with endobronchial valves [20], after LVRC ventilation is redistributed to the ipsilateral lobe, which is accompanied by an overall reduction in air trapping. In our study, we hypothesized that perfusion would respond in the same way. Indeed, one can hypothesize that reducing the vol- 
Fig. 2. a The graphs allow discriminating individual perfusion changes after LVRC in the 4 lung areas (red line: mean value for the entire cohort; color refers to the online version only). b Specific pulmonary perfusion in the areas adjacent to the treated ones (A), the ipsilateral untreated areas (B), the contralateral upper areas $(\mathrm{C})$, and the contralateral lower areas (D) before and after LVRC. The box plot depicts the median (line), 25th-75th percentile (box), and 5th-95th percentile (whiskers); the dots represent outliers.

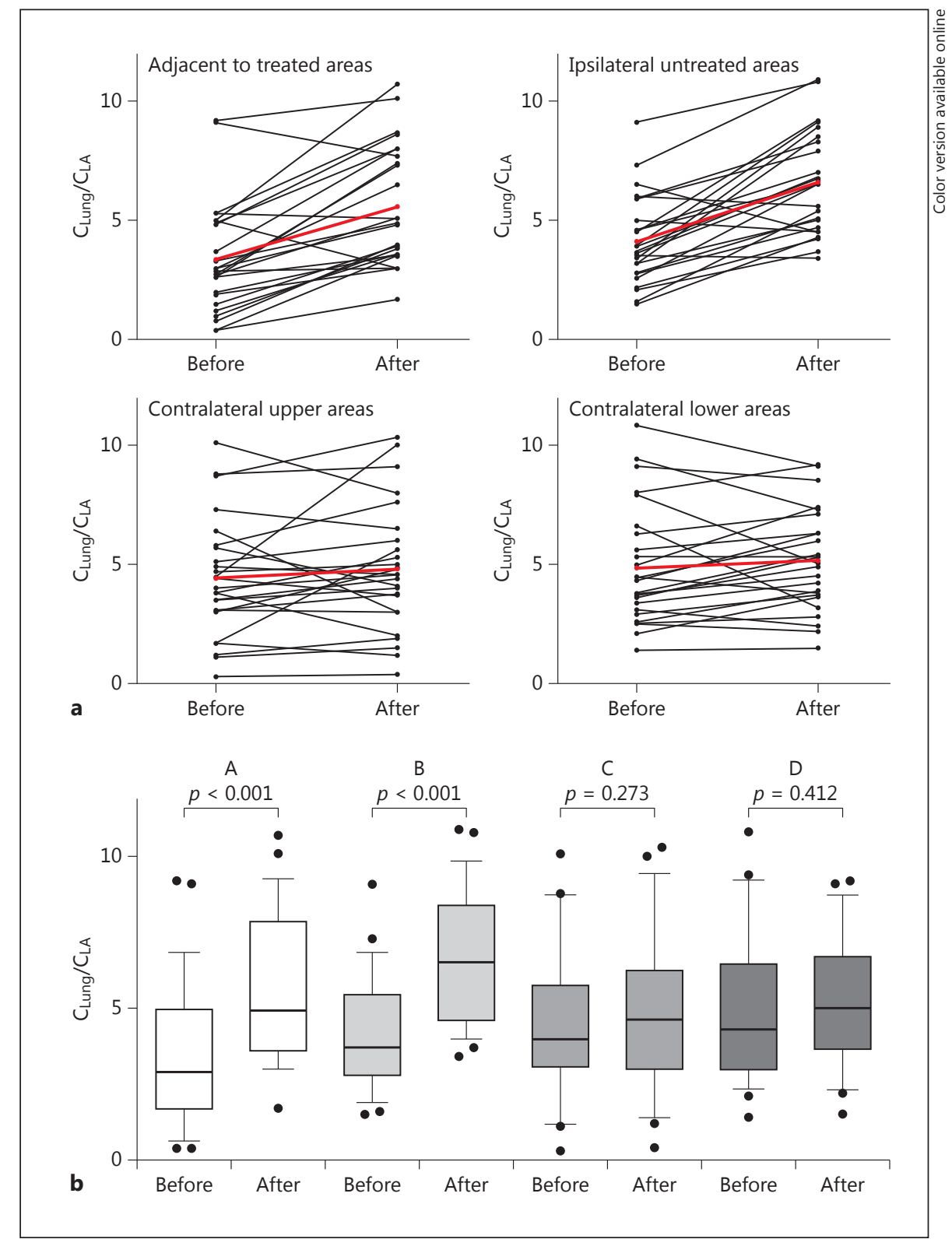

ume of the diseased lung may allow a significant blood flow redistribution from the treated lung towards the healthier adjacent lung parenchyma, as well as to the ipsilateral lobe. Therefore, we anticipated blood flow redistribution from the treated areas towards the untreated areas. This hypothesis is indeed consistent with our results, as the iodine concentration (representative of lung perfusion) increased by $61-65 \%$ in both the areas adjacent to the treated ones and the ipsilateral untreated areas after LVRC intervention. This could be explained by a better closure of vessels after coil placement in the emphysematous parenchyma, thus increasing the resistance to blood flow and facilitating its redirection from the treated parenchyma to regions with lower resistance.

Interestingly, we observed that perfusion was not significantly affected in the contralateral lobes after the LVRC procedure. Our results differ from those recently reported by Pizzaro et al. [21]. These authors studied the effect of valve therapy on lung perfusion and showed an increase in perfusion in the contralateral lung. It is also noteworthy that in this study, lung perfusion was as- 

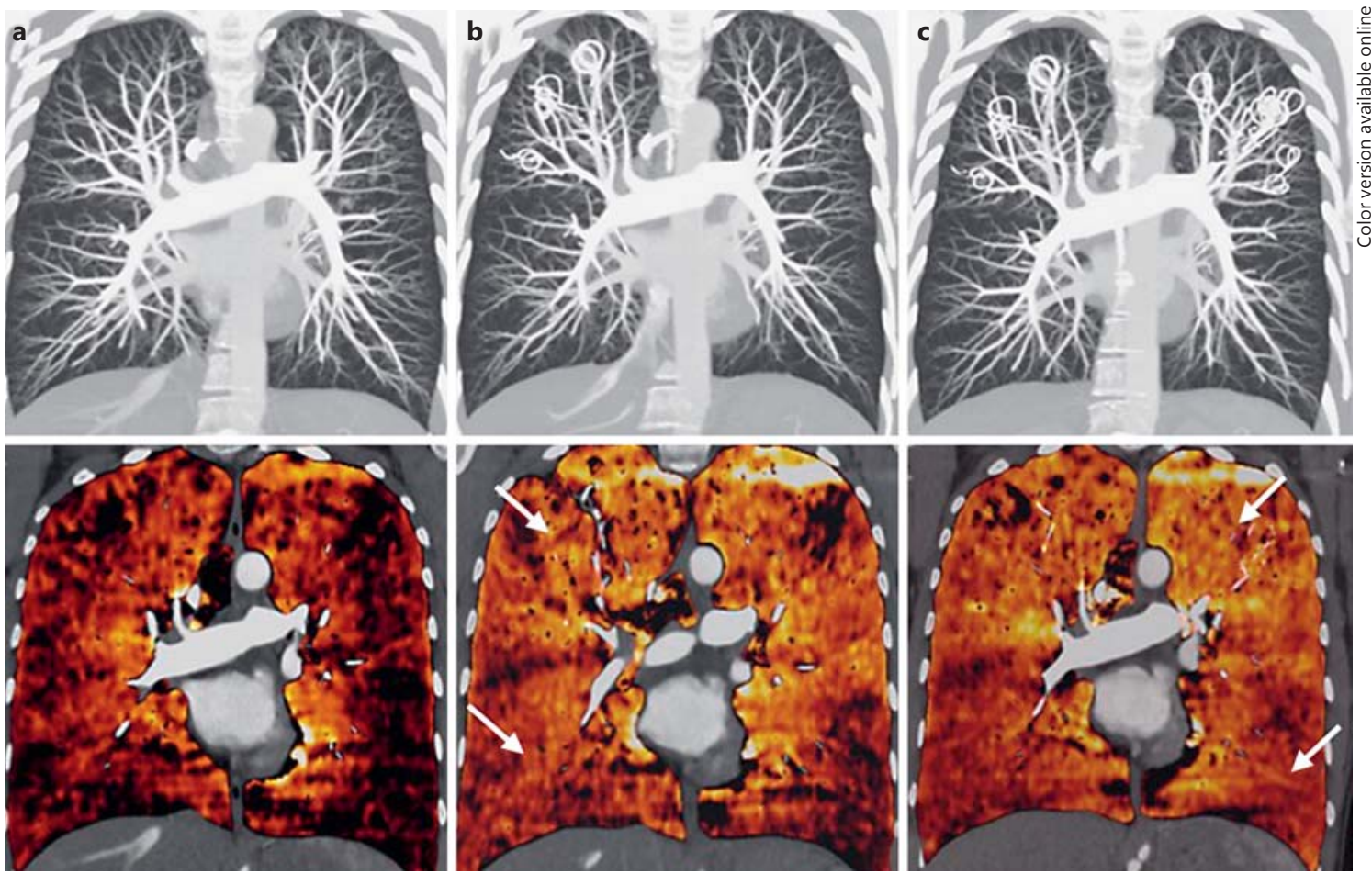

Fig. 3. Morphological coronal CT scans (upper panels) and the corresponding iodine perfusion maps (lower panels) before (a) as well as after the first LVRC on the right upper lobe (b) and after the second LVRC on the left upper lobe (c) in a 50-year-old woman. A significantly increased perfusion after the first procedure in

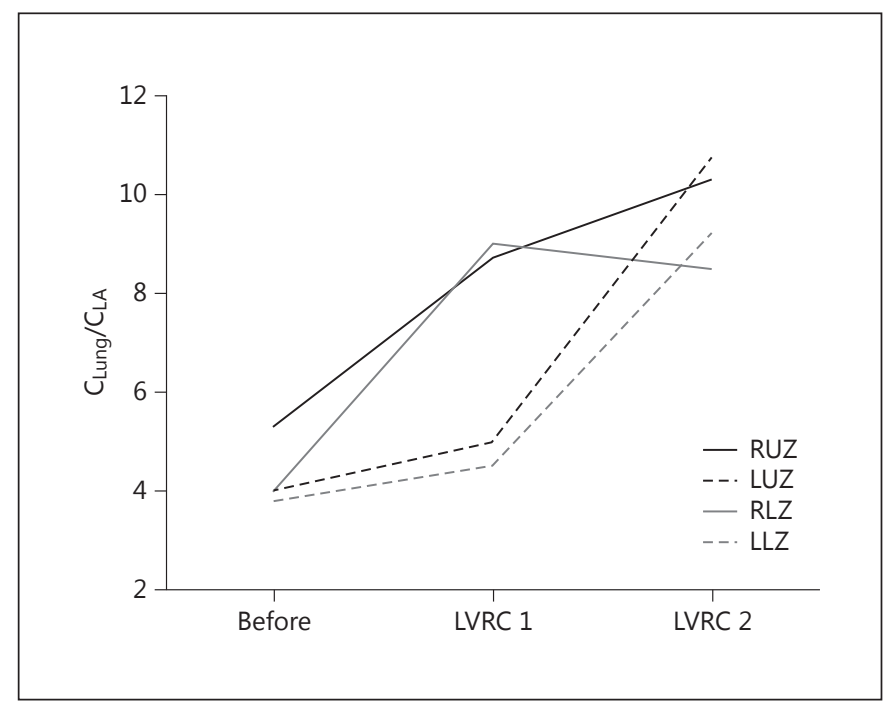

Fig. 4. Corresponding changes in the same patient for normalized lung parenchymal iodine concentration before and after the first (LVRC 1) and the second (LVRC 2) procedure. RUZ, right upper zone; LUZ, left upper zone; RLZ, right lower zone; LLZ, left lower zone. the right lung and a nonsignificantly increased perfusion in the left untreated lung were observed; afterwards, a significantly increased perfusion after the second procedure was observed in the left lung (arrows).

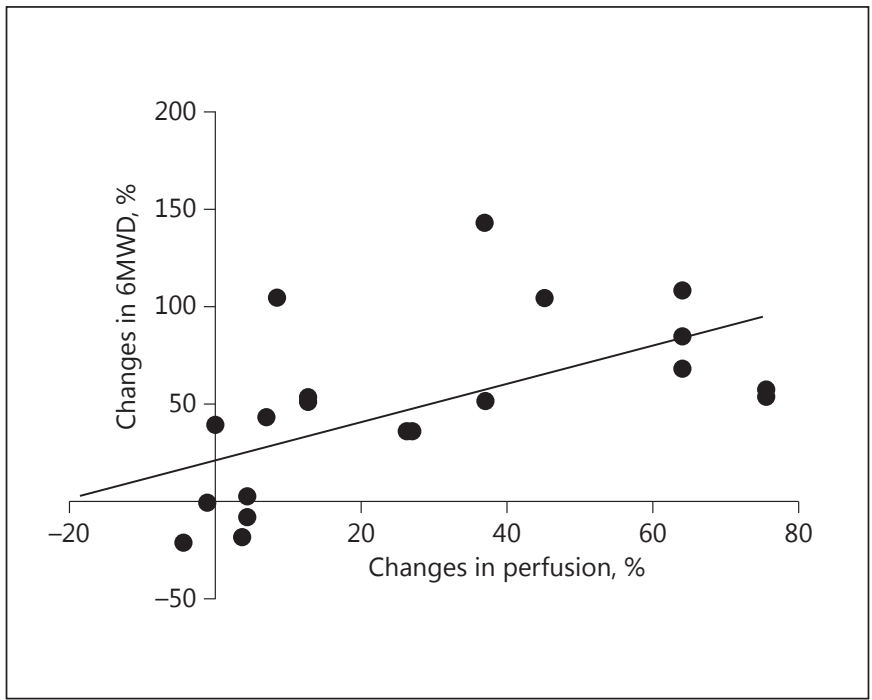

Fig. 5. Percent changes in global lung perfusion reported against percent changes in 6MWD. A significant correlation between increased 6MWD and increased perfusion was found $(p=0.0027$, $\left.R^{2}=0.3850\right)$. 
sessed by perfusion scintigraphy, which is a reference technique for the assessment of lung perfusion. Therefore, one might question the ability of contrast-enhanced DECT to accurately assess lung perfusion. As a matter of fact, several published works have shown that iodine perfusion obtained by contrast-enhanced DECT has an excellent correlation with the perfusion measured by pulmonary scintigraphy $[22,23]$. Several studies have also demonstrated that perfusion maps can be used to simply qualitatively and quantitatively assess areas of lung perfusion in a similar way to scintigraphy. Furthermore, the comparison between DECT and SPECT (single-photon emission CT) perfusion imaging seems to indicate a good correlation in the depiction of perfusion defects in pulmonary embolism or in chronic thromboembolic pulmonary hypertension [24]. Thieme et al. [25] showed that DECT had a sensitivity/specificity of $100 / 100 \%$ for pulmonary embolism, while the combination of SPECT and ventilation scintigraphy had a sensitivity/specificity of $85.7 / 87.5 \%$. Nowadays, many applications are available for routine clinical use, and, for example, perfusion maps generated by DECT are well established for evaluating acute pulmonary embolism [22, 23]. Moreover, and as opposed to ventilation/ perfusion scanning, DECT permits a combined morphological and functional analysis of the lung, usually obtained by a single acquisition with no extra radiation dose or extra intravenous iodine contrast medium injection. DECT permits a simultaneous assessment of the vascular anatomy, parenchymal morphology, and functional lung perfusion.

Interestingly, the improvement in pulmonary perfusion was significantly correlated with an improvement in exercise capacity, whereas pulmonary function test results tended to improve only slightly. Air trapping, represented by the RV and the RV/TLC ratio, tended to decrease after LVRC. The former was the only significantly improved pulmonary function test parameter, letting us suppose that the pulmonary ventilation/perfusion relationship has a tendency to improve, but that further improvement may be limited by a lack of improvement in pulmonary mechanics.

According to results obtained by others [26], endobronchial valves may improve right ventricular function, suggesting that the risk of developing or worsening pulmonary hypertension in those patients is low and one could consider this therapeutic approach in patients with slightly impaired hemodynamics. Our results may be in agreement with this assumption, as the significant improvement in $6 \mathrm{MWD}$ after treatment correlated with the overall increase in perfusion. However, this aspect was not specifically explored in our study.

The consequences of LVRC on lung mechanics and the ventilation/perfusion ratio may also be compared to the results obtained by surgical correction of pulmonary emphysema by LVRS. Here, some authors recently suggested an improvement in surrogate markers of endothelial function and systemic blood pressure in patients with severe emphysema [27]. In that study, it was concluded that the reduction in hyperinflation might play an independent role, providing a possible link between the severity of emphysema and endothelial dysfunction. Interestingly, a significantly decreased ventilation/perfusion inequality after LVRS was also outlined by Moonen et al. [28]. It seems unlikely that the very small improvement in lung mechanics provides a full explanation for the marked improvement in lung perfusion reported in our study. In fact, we showed a $65 \%$ increase in lung perfusion, while the best improvement in pulmonary function tests was reported for RV, with a less than $15 \%$ decrease. Thus other, undiscovered factors probably influence the effect of LVRC on parenchymal lung perfusion.

There are a few limitations of this study to be considered. The small sample size of 17 patients so far included - and subsequently the small number of LVRC procedures - forces us to interpret our results with caution. However, even though firm conclusions cannot be drawn from this pilot study, the initial results are very encouraging. The use of 2 different makes of CT scanners is another weakness of this study, which may make comparisons between the data more delicate. Nevertheless, this was counterbalanced by calibration of the iodine concentration in the lung by that in the left atrium, where patients underwent DECT using the same CT scanner. This procedure gave specific iodine concentrations that allowed us to use data from the 2 different devices. Potential sampling errors related to the drawing of ROIs are a further limitation. To minimize this limitation, multiple measurements were performed at standardized locations ( 6 at each of the 4 levels, corresponding to 24 set areas of ROI per patient) and the ROIs were made as large as possible, thereby avoiding artifacts, consolidations, or major vessels. Finally, the absence of a concomitant ventilation analysis could be a limitation; however, similar lung volumes at successive DECT acquisitions confirmed that the perfusion changes assessed by DECT were not influenced by variations in suspended inspiration during the acquisitions. 


\section{Conclusion}

Treatment of severe emphysema by LVRC induces a redistribution of blood flow, which allows some changes in pulmonary perfusion of the parenchyma adjacent to the treated lobe and of the ipsilateral lobe to significantly increase. Future studies in larger populations are warranted to confirm these results and to provide further insights concerning the changes induced by this treatment on the pulmonary ventilation/perfusion relationship.

\section{Authors' Contributions}

Conception and design: F.L., A.-L.H., J.P., X.M., and P.M.S.; analysis and interpretation: A.-L.H., F.L., O.H., and M.R.; drafting the manuscript for important intellectual content: F.L., A.-L.H., O.H., M.R., X.M., and P.M.S.

\section{Financial Disclosure and Conflicts of Interest}

None declared.

\section{Acknowledgements}

The authors would like to thank Dr. Catherine Torriani Hammon and Ms. Angela Frei for careful review of the manuscript and proofreading.

\section{References}

1 Kotloff RM, Thabut G: Lung transplantation. Am J Respir Crit Care Med 2011;184:159171.

- Inci I, Schuurmans MM, Boehler A, Weder W: Zurich University Hospital lung transplantation programme: update 2012. Swiss Med Wkly 2013;143:w13836.

-3 Naunheim KS, Wood DE, Krasna MJ, DeCamp MM Jr, Ginsburg ME, McKenna RJ Jr, Criner GJ, Hoffman EA, Sternberg AL, Deschamps C; National Emphysema Treatment Trial Research Group: Predictors of operative mortality and cardiopulmonary morbidity in the National Emphysema Treatment Trial. J Thorac Cardiovasc Surg 2006;131:43-53.

-4 Ramsey SD, Berry K, Etzioni R, Kaplan RM, Sullivan SD, Wood DE; National Emphysema Treatment Trial Research Group: Cost effectiveness of lung-volume-reduction surgery for patients with severe emphysema. N Engl J Med 2003;348:2092-2102.

5 Rugbjerg M, Iepsen UW, Jørgensen KJ, Lange P: Effectiveness of pulmonary rehabilitation in COPD with mild symptoms: a systematic review with meta-analyses. Int $\mathrm{J}$ Chron $\mathrm{Ob}$ struct Pulmon Dis 2015;10:791-801.

6 McDonald CF: ACP Journal Club. Review: pulmonary rehabilitation improves healthrelated QoL and exercise capacity more than usual care in COPD. Ann Intern Med 2015; 162:JC4.

-7 Shah PL, Zoumot Z, Singh S, Bicknell SR, Ross ET, Quiring J, Hopkinson NS, Kemp SV; RESET Trial Study Group: Endobronchial coils for the treatment of severe emphysema with hyperinflation (RESET): a randomised controlled trial. Lancet Respir Med 2013;1:233240 .
8 Deslee G, Klooster K, Hetzel M, Stanzel F, Kessler R, Marquette CH, Witt C, Blaas S, Gesierich W, Herth FJ, Hetzel J, van Rikxoort EM, Slebos DJ: Lung volume reduction coil treatment for patients with severe emphysema: a European multicentre trial. Thorax 2014;69:980-986

-9 Hartman JE, Klooster K, Gortzak K, ten Hacken NH, Slebos DJ: Long-term follow-up after bronchoscopic lung volume reduction treatment with coils in patients with severe emphysema. Respirology 2015;20:319-326.

10 Kontogianni K, Gerovasili V, Gompelmann D, Schuhmann M, Heussel CP, Herth FJ, Eberhardt R: Effectiveness of endobronchial coil treatment for lung volume reduction in patients with severe heterogeneous emphysema and bilateral incomplete fissures: a sixmonth follow-up. Respiration 2014;88:52-60.

-11 Zoumot Z, Kemp SV, Singh S, Bicknell SR, McNulty WH, Hopkinson NS, Ross ET, Shah PL: Endobronchial coils for severe emphysema are effective up to 12 months following treatment: medium term and cross-over results from a randomised controlled trial. PLoS One 2015;10:e122656

12 Johnson TR, Krauss B, Sedlmair M, Grasruck M, Bruder H, Morhard D, Fink C, Weckbach S, Lenhard M, Schmidt B, Flohr T, Reiser MF, Becker CR: Material differentiation by dual energy CT: initial experience. Eur Radiol 2007;17:1510-1517.

13 Ohana M, Jeung MY, Labani A, El Ghannudi $S$, Roy C: Thoracic dual energy CT: acquisition protocols, current applications and future developments. Diagn Interv Imaging 2014;95:1017-1026.
14 Thieme SF, Becker CR, Hacker M, Nikolaou K, Reiser MF, Johnson TR: Dual energy CT for the assessment of lung perfusion - correlation to scintigraphy. Eur J Radiol 2008;68:369-374.

15 Ameli-Renani S, Rahman F, Nair A, Ramsay L, Bacon JL, Weller A, Sokhi HK, Devaraj A, Madden B, Vlahos I: Dual-energy CT for imaging of pulmonary hypertension: challenges and opportunities. Radiographics 2014;34: 1769-1790.

16 Fujioka C, Horiguchi J, Kiguchi M, Yamamoto H, Kitagawa T, Ito K: Survey of aorta and coronary arteries with prospective ECG-triggered 100-kV 64-MDCT angiography. AJR Am J Roentgenol 2009;193:227-233.

17 Slebos DJ, Hartman JE, Klooster K, Blaas S, Deslee G, Gesierich W, Hetzel J, Hetzel M, McNulty W, Kemp SV, Kessler R, Leroy S, Stanzel F, Witt C, Zoumot Z, Herth FJ, Shah PL: Bronchoscopic coil treatment for patients with severe emphysema: a meta-analysis. Respiration 2015;90:136-145.

18 Gevenois PA, de Maertelaer V, De Vuyst P, Zanen J, Yernault JC: Comparison of computed density and macroscopic morphometry in pulmonary emphysema. Am J Respir Crit Care Med 1995; 152:653-657.

19 Gevenois PA, De Vuyst P, de Maertelaer V, Zanen J, Jacobovitz D, Cosio MG, Yernault JC: Comparison of computed density and microscopic morphometry in pulmonary emphysema. Am J Respir Crit Care Med 1996; 154:187-192.

20 Brown MS, Kim HJ, Abtin FG, Strange C, Galperin-Aizenberg M, Pais R, Da Costa IG, Ordookhani A, Chong D, Ni C, McNitt-Gray MF, Tashkin DP, Goldin JG: Emphysema lung lobe volume reduction: effects on the ipsilateral and contralateral lobes. Eur Radiol 2012;22:1547-1555. 
21 Pizarro C, Ahmadzadehfar H, Essler M, Tuleta I, Fimmers R, Nickenig G, Skowasch D: Effect of endobronchial valve therapy on pulmonary perfusion and ventilation distribution. PLoS One 2015;10:e0118976.

22 Pontana F, Faivre JB, Remy-Jardin M, Flohr T, Schmidt B, Tacelli N, Pansini V, Remy J: Lung perfusion with dual-energy multidetector-row CT (MDCT): feasibility for the evaluation of acute pulmonary embolism in 117 consecutive patients. Acad Radiol 2008; 15: 1494-1504.

-23 Sueyoshi E, Tsutsui S, Hayashida T, Ashizawa K, Sakamoto I, Uetani M: Quantification of lung perfusion blood volume (lung PBV) by dual-energy CT in patients with and without pulmonary embolism: preliminary results. Eur J Radiol 2011;80:e505-e509.
24 Nakazawa T, Watanabe Y, Hori Y, Kiso K, Higashi $\mathrm{M}$, Itoh $\mathrm{T}$, Naito $\mathrm{H}$ : Lung perfused blood volume images with dual-energy computed tomography for chronic thromboembolic pulmonary hypertension: correlation to scintigraphy with single-photon emission computed tomography. J Comput Assist Tomogr 2011;35:590-595.

25 Thieme SF, Graute V, Nikolaou K, Maxien D, Reiser MF, Hacker M, Johnson TR: Dual energy CT lung perfusion imaging - correlation with SPECT/CT. Eur J Radiol 2012;81:360365.

26 Pizarro C, Schueler R, Hammerstingl C, Tuleta I, Nickenig G, Skowasch D: Impact of endoscopic lung volume reduction on right ventricular myocardial function. PLoS One 2015; 10:e0121377.
27 Clarenbach CF, Sievi NA, Brock M, Schneiter D, Weder W, Kohler M: Lung volume reduction surgery and improvement of endothelial function and blood pressure in patients with chronic obstructive pulmonary disease. A randomized controlled trial. Am J Respir Crit Care Med 2015;192:307-314.

28 Moonen M, Xu J, Johansson A, Thylén A, Bake B: Effects of lung volume reduction surgery on distribution of ventilation and perfusion. Clin Physiol Funct Imaging 2005;25: 152-157. 